\title{
CONSIDERACIONES SOBRE LOS BENEFICIOS ECONÓMICOS DEL DISEÑO HABITABLE
}

\author{
Francisco Álvarez Partida*
}

\section{CONSIDERATIONS ON THE ECONOMIC

RESUMEN: A partir del concepto de habitabilidad, y en el marco de la sustentabilidad, se abordan las últimas consideraciones económicas nacionales y mundiales, tanto de instituciones como de académicos. Se hacen observaciones sobre las metodologías analizadas y se concluye sobre el caso mexicano, con miras a orientar a los interesados e influir en las políticas públicas.

PALABRAS ClAVE: Economía de la vivienda, habitabilidad, vivienda sustentable.
ABSTRACT: Based on the concept of habitability and the framework of sustainability, this article describes the state of art of the economic considerations observed at the global and national level by institutions and academics. Observations are made about the methodologies analyzed, and conclusions are drawn about the considerations that should be implemented in the Mexican scenario, with a view toward guiding the interested parties and influencing public policies.

KEYWORDS: habitability, sustainable housing, economics of housing.

* Profesor investigador del ITESO y doctorante ACEPTACIÓN: 5 de junio de 2018. en el posgrado de arquitectura de la UNAM. 


\section{CONSIDERACIONES SOBRE LOS BENEFICIOS ECONÓMICOS DEL DISEÑO HABITABLE}

\section{Introducción}

El problema de lo habitado, causado por una visión mercantilista, trajo como consecuencia graves crisis económicas y el abandono de centros urbanos y viviendas nuevas construidas en la periferia. Por ejemplo, en México, pese ha que hay un déficit habitacional, el INEGI contabilizó en el censo del 2010 cinco millones de viviendas deshabitadas, ${ }^{1}$ mientras que el Infonavit registra en todo el país 200 viviendas abandonadas $^{2}$ de

${ }^{1}$ INEGI, “Censo de Población y Vivienda 2010”, 2010, Instituto Nacional de Estadística y Geografia, en <http://www3.inegi.org.mx/sistemas/glosario/ Default.aspx?ClvGlo $=$ CPV2010\&s $=$ est $\& \mathrm{c}=27432>$.

${ }^{2}$ Everardo Martínez, "Infonavit recuperará 30 mil casas en 2016 ", El Financiero, $1^{\circ}$ de julio de 2016. las que financió. Es necesario volver a examinar el aspecto económico, dándole una nueva articulación con lo habitable y lo sustentable.

\section{Consideraciones económicas}

Para analizar el aspecto económico de la habitabilidad es necesario incluir ciertas variables que antes no se consideraban:

1) En la economía ambiental, se utiliza el concepto de externalidades, que son traslaciones de costos ambientales a la sociedad ${ }^{3}$ que

${ }^{3}$ Joan Martínez Alier y Jordi Roca Jusmet, Economía ecológica y política ambiental, 2013, México, FCE, p. 357. 
no se ven reflejados en la vivienda, aunque deberían.

2) De la misma manera, no se contabilizan costos sociales y culturales, como los efectos de no lograr la habitabilidad, que se verán más adelante.

3) También se omiten o se niegan ciertos beneficios económicos, como el uso de adobe y otros materiales económicos y accesibles, la autoconstrucción y la posibilidad de realizar actividades empresariales dentro de las viviendas.

Estas variables se influyen de manera recíproca; es decir, lo económico afecta a lo habitado y viceversa, lo habitado incide en lo económico. Se manejan diferentes escalas de análisis de lo habitado, como macro, meso o micro e inclusive interno. Se debe considerar todo el ciclo de vida de lo habitado. Las variables cambian por varias razones; por ejemplo, el desarrollo del país, el capital disponible o las políticas locales, así como los impactos de las otras dos dimensiones: la social (las diferencias demográficas de posición socioeconómica, edad, sexo, tipo de familia, etc.) y la ambiental (con diferentes condiciones de escasez o abundancia de agua, materiales de construcción, medios para obtener energía, biodiversidad y otros). Feifer $^{4}$

${ }^{4}$ Lone Feifer, "Sustainability indicator in buildings identifying key performance indicator", en afirma que se requiere una plataforma estandarizada para determinar la importancia relativa de todos estos elementos, lo cual se logra mediante el diálogo y el equilibrio entre las posiciones de los interesados. De otra manera, el análisis con que se seleccionan y ponderan las variables puede dar lugar a sesgos.

\section{El diseño habitable}

La función de habitar expresa una actividad relacionada con la ocupación de un espacio, una estructura o un conjunto de estructuras destinadas para este fin. ${ }^{5}$ La habitabilidad es el conjunto de características morfológicas y constructivas del espacio arquitectónico que hacen posible proporcionar las condiciones para el desempeño de las actividades de los individuos. Para este fin, es necesario que se satisfagan de forma integrada los requisitos de la compleja naturaleza humana, desde los más concretos —como los biológicos - hasta los más abstractos — como los estéticos y los éticos. ${ }^{6}$

PLEA 2011 Architecture and sustainable development, 2011, Bélgica, vol. 1, pp. 133-39.

${ }^{5}$ CONAVI, Código de edificación de la vivien$d a, 2010$, p. 31, en <http://www.cmic.org/comisiones/sectoriales/vivienda/biblioteca/archivos/ CEV\%20PDF.pdf>.

${ }^{6}$ Dulce María Barrios, "Caracterización de la vivienda sustentable", en Ester Maya y Eftychia Bournazotj (comps.), La vivienda en México. Temas contemporáneos, 2012, México, UNAM, p. 94. 
Los elementos de la habitabilidad son los siguientes:

1) El confort bioambiental, dado por la antropometría, la ergonomía y las condiciones climáticas, lumínicas, acústicas, olfativas y hápticas.

2) El confort psicológico, dado por la congruencia del mensaje enviado por el espacio arquitectónico, con la percepción y expectativas del usuario $;^{7}$ es decir, se genera un simbolismo.

3) El confort espiritual, dado por su esencia, que responde a la cultura propia de un lugar.

4) El confort estético, dado por la calidad estética del espacio arquitectónico que puede incluso compeler al acto humano perfecto o bien constituir "el derecho a pretender la belleza por sí misma". ${ }^{8}$

5) El confort social, dado al tener un espacio territorial y refugio higiénico y sano para sus moradores, donde se pueda socializar. ${ }^{9}$

6) Solidez y seguridad, dadas por el uso de materiales y procedimientos de construcción adecuados. $^{10}$

${ }^{7}$ Loc. cit.

${ }^{8}$ Le Corbusier, La casa de los hombres, 2008, Barcelona, Apóstrofe, p.134.

${ }^{9}$ Barrios, op. cit.

${ }^{10}$ Ibid., p. 95.

\section{Liga con la sustentabilidad}

Varios autores dedican sus reflexiones sobre el acto de habitar al sitio habitado, el planeta Tierra. Heidegger ${ }^{11}$ dice que la palabra alemana para habitar (wohnen) está relacionada con el término gótico wunian, que significa "estar en paz, haber alcanzado la paz". La palabra Friede (paz) significa lo libre (freie), lo "protegido contra daño y amenaza, salvaguardado [...], es decir preservado". Lo que debemos de preservar es la Tierra, ya que solo podemos habitarla si está en condiciones de ser habitada.

Por su parte, Leonardo Boff escribe que "la Tierra no está ante nosotros como algo distinto de nosotros mismos. Tenemos la Tierra dentro de nosotros mismos. El vocablo hombre viene de humus [...] Somos la Tierra, [...] en su momento de autorrealización y autoconciencia. Formamos una misma realidad, diversa, compleja y única". ${ }^{12}$ Está visión de los pueblos del sur, más latinoamericana, que se conoce como "ecologismo de los pobres", ${ }^{13}$ nos vincula con nuestro hábitat y nos hace sentirnos parte de él.

\footnotetext{
${ }^{11}$ Martin Heidegger, Construir, habitar, pensar, 1985, Córdoba, Alción, trad. de Ana Carlota Gebhardt.

${ }^{12}$ Leonardo Boff, Cuidar la tierra. Hacia una ética universal, 2001, México, Dabar.

${ }^{13}$ Joan Martínez Alier, El ecologismo de los pobres: conflictos ambientales y lenguajes de valoración, 2005, Barcelona, Icaria.
} 
Con lo anterior llegamos al paradigma de la sustentabilidad, definido como la manera de proporcionar buen vivir, calidad de vida de la gente, dentro de los medios de la naturaleza, de manera justa y equitativa para toda la humanidad, las otras especies y las generaciones futuras. Lo sustentable se apoya en tres pilares principales, el ambiental, el sociocultural y el económico, siguiendo el modelo de la "triple cuenta de resultados" de Elkington. ${ }^{14}$ Con estos pilares se busca alcanzar lo siguiente:

1) Que no se consuman los recursos más rápidamente de lo que la naturaleza puede renovarlos ni se produzcan deshechos más deprisa de lo que pueden ser absorbidos.

2) Que todos los seres humanos tengan la posibilidad de alcanzar el mismo nivel de desarrollo, que vivan o estén bien o sean felices, y que puedan participar en las decisiones para lograrlo, con respeto a sus formas de vida y cosmovisiones particulares.

3) Lo viable, una economía que genere el mayor bienestar posible para todos.

${ }^{14}$ Dalma Berkovics, Cannibals with fork. The triple bottom line of 21st century business, 2010, Capstone Publishing, Mankato.
Consideraciones del beneficio económico en el diseño habitable

El programa de las Naciones Unidas para el Hábitat ${ }^{15}$ considera los siguientes aspectos económicos de la vivienda en países en vías de desarrollo.

1) Facilitar la adquisición de la vivienda para diferentes grupos sociales.

-Regulaciones e incentivos para los mercados de construcción y bienes raíces.

-Provisión de subsidios y otros tipos de asistencia para la vivienda. -Desarrollo de un mercado de hipotecas asequibles.

-Provisión de suelo e infraestructura públicos para proyectos de vivienda.

-Construcción de vivienda pública y estímulos para las comunidades de base, las cooperativas y los programas de vivienda de renta y no lucrativos.

-Regulación y estimulación de la autoconstrucción y las mejoras de la vivienda.

-Modificación de los códigos de construcción, las regulaciones

${ }^{15} \mathrm{UN}-H a b i t a t$, Sustainable housing for sustainable cities: a policy framework for developing countries (United Nation Human Settlements Programme 2012), en <http://peoplebuildingbettercities. org/wp-content/uploads/2013/06/SustainableHousing-Policy-Framwork.pdf $>$. 
y los procedimientos para reducir los costos de la vivienda.

-Asociaciones entre gobiernos centrales y locales, compañías privadas y otros interesados para realizar proyectos de vivienda en comunidades locales.

-Reforzamiento de la industria de la construcción de la vivienda y los mercados de materiales de construcción asequibles.

2) Asegurar mercados de vivienda equilibrados con opciones de tenencia asequibles.

-Propiedad privada. Con políticas macroeconómicas que favorecen excesivamente el mercado y la propiedad de vivienda, lo que contribuye a la volatilidad del mercado.

-En renta.

-Vivienda cedida por servicios, de ONG y otras.

3) Reconocer la edificación de vivienda asequible como una fuente de empleo.

-Trabajo en la vivienda o en las cercanías.

-Estímulos al desarrollo de las pequeñas empresas.

-Uso de tecnologías intermedias de bajo costo.

-Autoconstrucción como la única opción para personas con escasos recursos económicos.

4) Ayudar a las empresas domésticas. Aunque estén ligadas a la infor- malidad, es más eficaz reconocerlas y apoyar su formalización gradual, antes que ignorarlas o dañarlas.

-Tibaijuka ${ }^{16}$ proporciona los siguientes datos sobre empresas domésticas: en Buenos Aires, 8\% de los trabajadores del sector manufacturero trabajan en su hogar. En Venezuela, $45 \%$ de toda la industria del vestido es domiciliaria. En Zimbabue, 77\% de todas las empresas están en los hogares.

5) Movilizar los ahorros y las finanzas domésticas.

-Remesas.

-Microfinanciamiento.

-Combinación prácticas formales e informales de financiamiento. -Administración y mantenimiento de la vivienda.

-Fomento de la resiliencia y las pruebas del funcionamiento futuro de las viviendas.

Hay varios sistemas de evaluación de la sustentabilidad de las edificaciones (SESE), los cuales abarcan los aspectos ambientales, socioculturales $\mathrm{y}$ económicos, aunque esta enunciación tiene otras categorías, como el sitio, el agua, la energía, los residuos, etc. El IHOBE $^{17}$ hizo una com-

${ }^{16}$ Anna K. Tibaijuka, Building prosperity: Housing and economic development, 2009, Londres, Earthscan.

${ }^{17}$ IHOBE, Green building rating systems: ¿Cómo evaluar la sostenibilidad de la edificación?, 2010. 
paración entre los principales SESE y definió un conjunto de variables económicas, con temas y subtemas, y definió un propósito de evaluación.

Los sistemas comparados fueron el Código del Gobierno Británico para las viviendas sostenibles (BREEAM), Liderazgo en Diseño Ambiental y Energético (LEED) de los Estados Unidos, el CASBEE de Japón, los Green Globes de Canadá y Estados Unidos, la Green Star de Australia, la Alta
Calidad Medioambiental HQE) de Francia, y las guías de edificación sostenible del País Vasco. Para la dimensión económica utilizaron tres categorías: Efectos colaterales y externalidades; Financiación y Gestión, y Valor de ciclo de vida.

Como se observa en la Tabla 1, el sistema que comprende más variables del aspecto económico de la sustentabilidad es el HQE, y los que menos, BREEAM y Green Star.

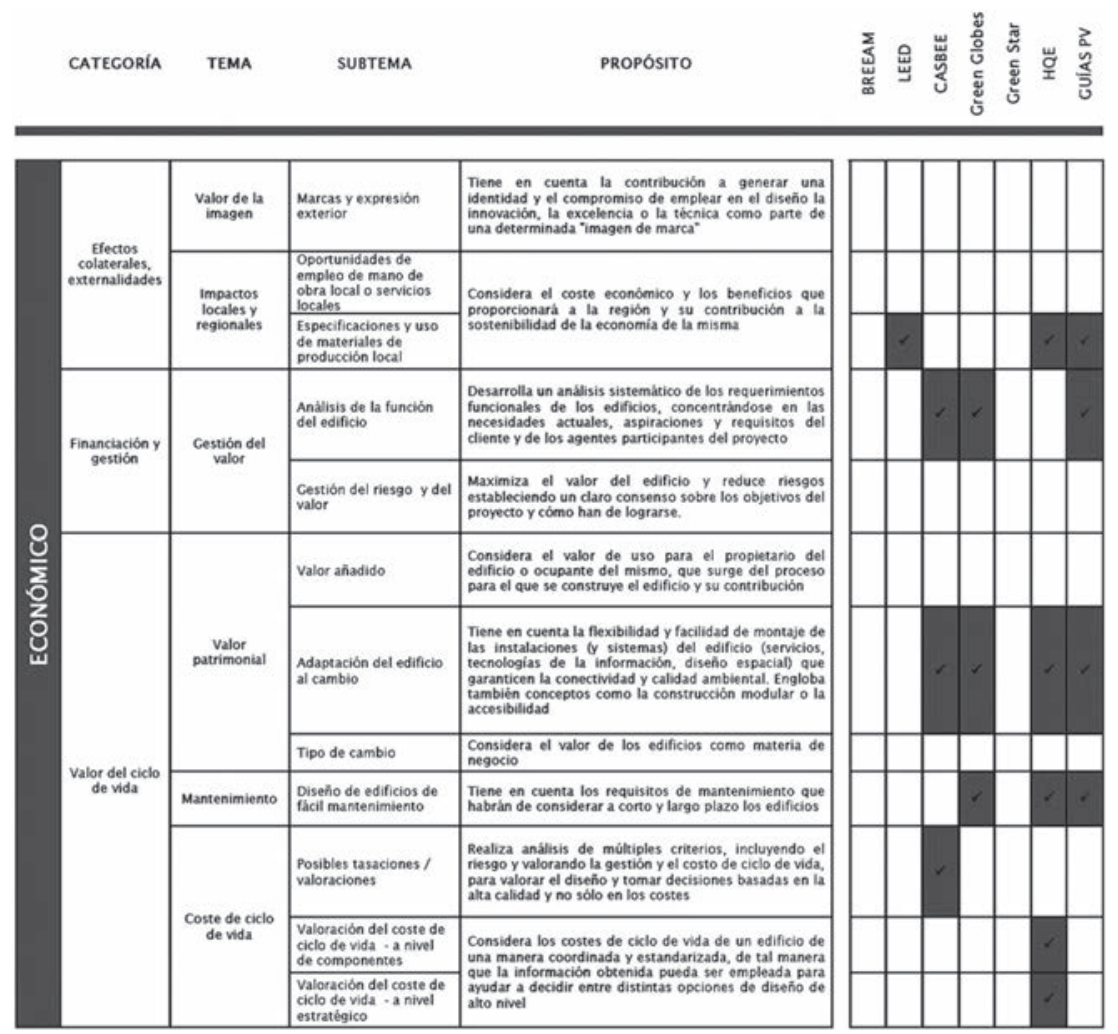

Tabla 1. Variables económicas en los sistemas de evaluación de la sustentabilidad de las edificaciones Fuente: IHOBE, Green building rating systems:

¿Cómo evaluar la sostenibilidad de la edificación? 
Gibberd ${ }^{18}$ realizó un análisis de los criterios de sostenibilidad para aplicarse en edificaciones de países en vías de desarrollo, en el que tomó los siguientes aspectos para evaluar el desempeño económico de las edificaciones.

\section{1) Economía local. La construcción} y la administración de los edificios pueden tener un gran impacto en la economía de una localidad, pues la fomentan al aprovechar y desarrollar habilidades y recursos locales.

- Contratistas locales.

-Suministro de materiales de construcción locales.

-Componentes, accesorios y mobiliario de manufactura local.

-Oportunidades de subcontratos para pequeñas empresas emergentes.

-Mantenimiento y reparaciones llevadas a cabo por los usuarios o por contratistas locales.

2) Eficiencia de uso. Los edificios cuestan dinero y consumen recursos, sean utilizados o no. El uso eficaz y eficiente de los edificios apoya la sostenibilidad porque se reducen los desperdicios y la necesidad de levantar edificaciones adicionales.

${ }^{18}$ Jeremy Gibberd, "Integrating sustainable development into briefing and design processes of building in developing countries: An assessment tool", 2003, Pretoria, University of Pretoria.
-Buen uso del espacio.

-Administración eficiente del espacio.

-Uso compartido de las instalaciones.

-Acceso a las tecnologías de comunicación.

-Horario eficiente de ocupación.

3) Adaptabilidady flexibilidad. Casi todos los edificios tienen una expectativa de vida de al menos 50 años. Es probable que el uso de los edificios cambie. Los edificios que se pueden adaptar fácilmente al cambio, apoyan la sostenibilidad, porque aminoran los requisitos y los costos de la adaptación física, el consumo de energía, así como la necesidad de nuevos edificios.

-Espacios listos para adaptarlos a diferentes usos.

-Facilidad de reconfiguración del mobiliario.

-Servicios configurados para aumentarlos o modificarlos fácilmente.

-Estructura configurada para variar la distribución interna.

-Circulaciones verticales y servicios básicos configurados para permitir diferentes arreglos espaciales.

4) Costos corrientes. Si se tiene en cuenta la vida media de las edificaciones, habitarlas y mantenerlas cuesta dinero y recursos. El uso 
eficaz y eficiente de los edificios apoya la sostenibilidad porque se reducen los desperdicios y la necesidad de más edificaciones.

-Se entienden y se consideran las necesidades de mantenimiento corriente de los edificios.

-Los edificios se mantienen fácilmente y sin peligro.

-Los edificios son seguros sin requerir grandes costos corrientes. -Los costos corrientes de agua, energía y seguros son mínimos. -Los ocupantes son conscientes de los niveles de consumo y producción de desperdicios.

5) Costo de capital. En general, las edificaciones son de los activos más valiosos que la gente, las organizaciones y el gobierno poseen. El dinero gastado no está disponible para otros rubros, como la salud, la educación y el desarrollo de negocios. A menudo, el costo de las edificaciones aumenta los precios del alojamiento y los servicios, de modo que quedan fuera del alcance de la gente con recursos limitados. -Provisión de incentivos a los consultores, para reducir el capital y los costos corrientes.

-Diseño costeable para minimizar desperdicios.

-Costos iniciales de construcción limitados.
-Minimización de costos de capital mediante acuerdos entre usuarios y asociados.

-Reducción de la cantidad de nuevos espacios por medio de acuerdos para compartir los espacios.

\section{El caso mexicano}

En México, la estrategia nacional para la vivienda sustentable ${ }^{19}$ define los componentes económicos y estipula que

están relacionados con los costos y beneficios económicos asociados a la vivienda, sus servicios y su ubicación, menciona que la vivienda constituye un factor fundamental de la estabilidad económica de las familias y uno de los principales amortiguadores frente a momentos de estrés económico — cuando la compra no representa un costo financiero que supera la capacidad de las familias-. La plusvalía de la vivienda en el mediano y largo plazo puede convertirse también en uno de los principales activos económicos de las personas. En los hogares mexicanos existe un gasto importante en los consumos de agua, gas y energía eléctrica así como en el transporte. La disminución de los consumos de estos servicios puede significar beneficios económicos importantes para los hogares.

${ }^{19}$ Conavi, Estrategia nacional para la vivienda sustentable. Componente ambiental de la sustentabilidad, 2013. 
En concordancia con lo anterior, el Centro Mario Molina ${ }^{20}$ considera seis categorías de impacto económico (Tabla 2): variación en el gasto familiar, ahorros por la implementación de ecotecnologías; gastos de vivienda como porcentaje del ingreso, gastos de transporte como porcentaje del ingreso, formación de patrimonio y plusvalía.

\section{Conclusiones}

Para alcanzar una habitabilidad adecuada, se deben revisar variables económicas que actualmente se omiten y darle valor a los elementos sociales y ambientales. Los aspectos económicos, ambientales y sociales conforman el paradigma de la sustentabilidad. El aspecto ambiental implica que lo habitado esté dentro de los límites de la capacidad del planeta y que su huella ecológica sea pequeña. El concepto de habitabilidad apunta a lograr el confort bioambiental, psicológico, espiritual, estético y social y a dar seguridad a los ocupantes. La habitabilidad forma parte del aspecto social.
Lo habitado ejerce una influencia recíproca con lo económico y lo ambiental, se puede manejar a diferentes escalas y se debe considerar todo su ciclo de vida. Las consideraciones económicas y su importancia varían con los lugares y deben ponderarse en procesos participativos y de diálogo, partiendo de una plataforma uniforme.

Tradicionalmente se ha dejado a un lado el impulso a otras formas de tenencia de lo habitado que fomenten la economía local, permitan una mezcla adecuada de los usos urbanos y favorezcan las empresas domésticas. La adaptabilidad y la flexibilidad del hábitat consideran variables que están en cambio, como la conformación de las familias o la pirámide de edades.

Se deben promover los microfinanciamientos, combinados con prácticas formales y el fondeo colectivo, con el fin de facultar a los usuarios de bajos recursos e impulsar el desarrollo de este hábitat. También se debe facilitar la autoconstrucción y la construcción con materiales y sistemas alternativos. Todo lo anterior contribuirá a tener una mejor vivienda y a que haya menos abandonos.

${ }^{20}$ Evaluación de la sustentabilidad de la vivienda en México, síntesis, 2012, México Centro Mario Molina. 


\begin{tabular}{|c|c|c|}
\hline Índice & Indicador & Evaluación del desempeño \\
\hline \multirow{6}{*}{ Económico } & $\begin{array}{l}\text { Variación en el gasto } \\
\text { familiar }\end{array}$ & $\begin{array}{l}\text { Número de usuarios que perciben un aumento, disminución o } \\
\text { constancia en sus gastos a raiz del cambio de vivienda. } \\
\text { Incluye gastos en: vivienda, agua, luz, gas, alimentación, } \\
\text { transporte, educación, salud y esparcimiento. }\end{array}$ \\
\hline & $\begin{array}{l}\text { Ahorros por la } \\
\text { implementación de } \\
\text { ecotecnologias }\end{array}$ & $\begin{array}{l}\text { Número de usuarios con ecotecnologías que perciben o no } \\
\text { ahorros en sus gastos de luz, agua o gas. }\end{array}$ \\
\hline & $\begin{array}{l}\text { Gastos de vivienda } \\
\text { como porcentaje del } \\
\text { ingreso }\end{array}$ & $\begin{array}{l}\text { Porcentaje del ingreso que los usuarios dedican a sus gastos de } \\
\text { vivienda. }\end{array}$ \\
\hline & $\begin{array}{l}\text { Gastos de transporte } \\
\text { como porcentaje del } \\
\text { ingreso }\end{array}$ & $\begin{array}{l}\text { Porcentaje del ingreso que los usuarios dedican a sus gastos de } \\
\text { transporte. }\end{array}$ \\
\hline & $\begin{array}{l}\begin{array}{l}\text { Formación de } \\
\text { patrimonio }\end{array} \\
\end{array}$ & $\begin{array}{l}\text { Número de usuarios que son dueños de la vivienda o la están } \\
\text { pagando mediante un crédito hipotecario. }\end{array}$ \\
\hline & Plusvalia & $\begin{array}{l}\text { Cálculo complejo de un índice de plusvalia por vivienda. } \\
\text { Incluye: satisfacción respecto a caracteristicas de la vivienda y su } \\
\text { entorno, datos de la base de avalúos de SHF, asi como la } \\
\text { calificación del municipio en el INCOMUV. }\end{array}$ \\
\hline
\end{tabular}

Tabla 2. Indicadores económicos y forma de evaluación.

Fuente: Evaluación de la sustentabilidad de la vivienda en México. 
Se prohíbe su reproducción total o parcial por cualquier medio, incluido electrónico, sin permiso previo y por escrito de los editores. 